\title{
Morphology of three polycentric rumen fungi and description of a procedure for the induction of zoosporogenesis and release of zoospores in cultures
}

\author{
Y. W. $\mathrm{Ho}^{1 *}$ AND T. BAUCHOP ${ }^{2}$ \\ ${ }^{1}$ Department of Biology, Faculty of Science and Environmental Studies, Universiti Pertanian Malaysia, 43400 Serdang, \\ Selangor Darul Ehsan, Malaysia \\ ${ }^{2}$ Department of Biochemistry, Microbiology and Nutrition, The University of New England, Armidale, NSW 2351, \\ Australia
}

(Received 22 May 1990; revised 3 September 1990; accepted 26 September 1990)

\begin{abstract}
Three polycentric rumen fungi, LL, LC2 and Ruminomyces elegans (C2), isolated from the rumen of cattle were grown in six culture media. LL and LC2 were morphologically similar. Their characteristics resembled those of Orpinomyces and Neocallimastix joyonii, and they grew well and produced sporangia after $3-4 \mathrm{~d}$ growth in all the media. R. elegans differed morphologically from LL and LC2, but although it also grew well in all media, abundant sporangia occurred only after 2-3 d growth in media containing cellulose. Undifferentiated sporangia were produced by all three isolates; differentiation of the sporangia did not occur in the spent growth media. However, if thalli possessing recently-formed sporangia were transferred to, or flooded with, fresh liquid medium or rumen fluid, zoosporogenesis and liberation of zoospores occurred within 17-20 min for isolates LL and LC2 and 30 min for $\boldsymbol{R}$. elegans. Procedures for inducing zoosporogenesis by polycentric anaerobic fungi are described.
\end{abstract}

\section{Introduction}

In early work on the gut anaerobic fungi of herbivores (Orpin, 1975, 1976, 1977a; Bauchop, 1979a, b, 1989), the fungi described all had monocentric thalli (one sporangium per thallus), and the species which were classified formally were placed within the order Spizellomycetales in the Class Chytridiomycetes (Heath et al., 1983). More recently, a different group of anaerobic rumen fungi possessing polycentric thalli (several sporangia per thallus) has been described (Akin \& Rigsby, 1987). Akin et al. (1988) reported three distinct polycentric isolates based on sporangial variations. Phillips (1988) also described the morphologies of three apparently different polycentric rumen fungi: one of these did not produce any zoospores under the culture conditions used, although sporangia were formed abundantly; the other two produced zoospores only infrequently. Subsequently, a polycentric species with polyflagellate zoospores, Orpinomyces bovis (Barr et al., 1989), was formally described and assigned its taxonomic position. Breton et al. (1989) described a polycentric fungus with polyflagellate zoospores and named it Neocallimastix joyonii. These taxonomic discrepancies will require resolution. Another polycentric fungus $(\mathrm{C} 2)$ which produces monoflagellate zoospores has recently formally been described and assigned to a new genus and species - Ruminomyces elegans (Ho et al., 1990).

Unlike the monocentric rumen fungi, which form sporangia and release zoospores in a natural and regular sequence, a common characteristic of axenic cultures of the polycentric anaerobic rumen fungi is the paucity or absence of zoosporogenesis. Although some polycentric isolates form sporangia readily and abundantly in certain media, the majority of the sporangia do not differentiate and release zoospores. As the ultrastructure of the mature zoospore is a key diagnostic characteristic used in Chytridiomycetes taxonomy, it is not possible to study the life cycle or the taxonomy of these fungi unless zoospores are obtained easily and abundantly.

A rapid and simple procedure for inducing zoosporogenesis and obtaining abundant zoospores from three polycentric anaerobic rumen fungi LL, LC2 and Ruminomyces elegans $(\mathrm{C} 2)$ is described in this paper.

\section{Methods}

Animals. The cattle (Bos taurus) from which the fungi were isolated were 18-month-old Hereford steers fed oaten chaff once daily. 
Isolation of fungi. Polycentric anaerobic fungi LL, LC2 and R. elegans (C2) were isolated by serial dilution of strained bovine rumen contents containing $15 \%(\mathrm{v} / \mathrm{v})$ clarified rumen fluid in roll tubes. Cellulose agar medium (CAM) was modified from that previously described (Bauchop, 1979a) by the addition of $1.5 \%(w / v)$ agar (Difco) and the replacement of glucose by ball-milled Whatman no. 1 filter paper $(0.2 \%$ w/v). Tubes of molten CAM were maintained at $46^{\circ} \mathrm{C}$ in a waterbath and an antibiotic mixture $(5 \%, \mathrm{v} / \mathrm{v}$; see below) was added immediately before inoculation. Rolled tubes were incubated at $39^{\circ} \mathrm{C}$, and by $48 \mathrm{~h}$ a number of large fungal colonies had developed. Polycentric fungi were distinguished from monocentric forms on the basis of their ability to spread out into the cellulose agar. All forms exhibited cellulose digestion. Colonies were picked and transferred to glucose sloppy medium (GSM) containing antibiotics (Bauchop, 1979a). Reinoculation into antibiotic-free roll tubes and re-isolation into GSM produced pure cultures which were designated LL, LC2 and $R$. elegans (C2).

As the fungi did not produce abundant zoospores under normal culture conditions, after the means of obtaining abundant zoospore production had been discovered, the fungi were again re-isolated in dilution series to ensure that the organisms isolated were derived from single zoospores. The fresh isolates appeared identical to the parent cultures.

The fungi were transferred to cellulose sloppy medium (CSM), which was CAM with agar reduced to $0.1 \%(w / v)$. The organisms were maintained by transfer every $4-5 \mathrm{~d}$.

Antibiotic mixture. This contained $1 \cdot 212 \%(\mathrm{w} / \mathrm{v})$ benzylpenicillin, $0.265 \%(\mathrm{w} / \mathrm{v})$ streptomycin sulphate and $0.060 \%(\mathrm{w} / \mathrm{v})$ chloramphenicol. The antibiotics were dissolved in distilled water that had been freshly boiled and cooled under an atmosphere of $\mathrm{CO}_{2}$, and were dispensed under an atmosphere of $\mathrm{CO}_{2}$ into serum bottles, which were stoppered and sealed. For isolation of fungi from rumen fluid, $5 \%(\mathrm{v} / \mathrm{v})$ of antibiotic mixture was added.

Culture conditions. The anaerobic techniques described by Hungate (1969) were used for the maintenance and subculturing of fungal cultures. Cultures were grown anaerobically at $39^{\circ} \mathrm{C}$ in tubes containing $7.0 \mathrm{ml}$ medium. Media were inoculated by transferring $5 \%$ of the volume of 4-5 d-old cultures.

In the experimental studies, six different media were used, four liquid and two solid. Media CSM, GSM and CAM have been described above. Other media were modifications of GSM. Glucose agar medium (GAM) had an increased agar content $(1.5 \%$, w/v) or alternatively the agar was omitted and glucose replaced by $0.3 \%(w / v)$ cellobiose (cellobiose broth, CELB) or 10 pieces of wheat straw leaf $5-6 \mathrm{~mm}$ in length (wheat straw broth, WSB).

Tubes with fungal colonies growing in liquid media were shaken vigorously by hand for 20-30 s daily to ensure that the colonies did not grow into compact clumps but were broken up and grew in loose mycelial mats. The fungal colonies growing in the various media were monitored daily for sporangium development using a dissecting microscope.

Pieces of mycelial mats which had produced abundant undifferentiated sporangia in liquid media were selected using a sterile hypodermic syringe and transferred to fresh liquid medium. Culture tubes were reincubated at $39^{\circ} \mathrm{C}$ and the sporangia were observed periodically for zoosporogenesis and release of zoospores. With solid media, colonies with undifferentiated sporangia were flooded with fresh liquid media such as GSM or CELB, or with clarified rumen fluid (filtered and centrifuged twice at $15000 \mathrm{~g}$ ), and were observed for zoosporogenesis and zoospore liberation.

The fungi were monitored in the culture tubes using a dissecting microscope, and examined in detail, at higher magnification, using bright-field and phase-contrast microscopy.

\section{Results and Discussion}

The general morphological characteristics of $L L$ and LC2 grown on the different culture media were similar, but those of $R$. elegans were distinctly different.

Both LL and LC2 produced large rhizomycelia comprising extensively branched hyphae which could be tubular and uniform in diameter or very wide and irregular, sometimes with constrictions. Sporangiophores usually developed from the wider hyphae and they initially developed as small irregular-shaped pegs which later elongated to form tubular sporangiophores (Fig. 1a). Many sporangiophores could be formed from one hypha and they could be both intercalary and terminal. Both fungal isolates grew well and developed abundant sporangia in all the media. Growth in agar roll tubes of CAM resulted in large cleared zones around the colonies. Development of sporangia usually occurred after 3-4 d growth in all the media. Colonies grown in tubes which were shaken daily produced sporangia earlier ( $3 \mathrm{~d})$ and more abundantly than those grown in unshaken tubes. The sporangia produced were usually spherical (Fig. 1a). Although sporangia were produced abundantly, most did not differentiate to form zoospores. Thus, zoospores were rarely observed in cultures; the few zoospores discovered were polyflagellate (Fig. $1 b$ ). The morphological characteristics of LL and LC2 appeared similar to those of Orpinomyces (Barr et al., 1989) and Neocallimastix joyonii (Breton et al., 1989). Although LL and LC2 seem to be morphologically similar, it is not possible at this stage to acertain whether they belong to the same species.

$R$. elegans produced a much smaller but more compact rhizomycelium than LL and LC2. The hyphae were highly branched and many of the thicker ones were constricted at intervals, giving the hyphae a bead- or sausage-like appearance (Fig. 2). Frequently, small lobed or disc-like structures (Fig. 2) were produced by the hyphae. These structures were very similar to the 'appressoria' produced by the anaerobic rumen fungi from cattle and swamp buffalo described by Ho et al. (1988a). A detailed description of the morphological characteristics of $R$. elegans has been given by Ho et al. (1990). R. elegans grew well in all media tested but only developed abundant sporangia in CSM, CAM and WSB. Sporangia were formed abundantly after $2-3 \mathrm{~d}$ growth in these three media. It appeared that $R$. elegans, unlike LL and LC2, required cellulose as substrate for abundant sporangia development. The sporangia were mainly fusiform with an acuminate tip (Fig. 3a), but occasionally spherical sporangia were formed. As with LL and $\mathrm{LC} 2$, the sporangia produced were mostly undifferentiated. On the few occasions that zoospores were found, they were monoflagellate (Fig. $3 b$ ). A polycentric fungus 

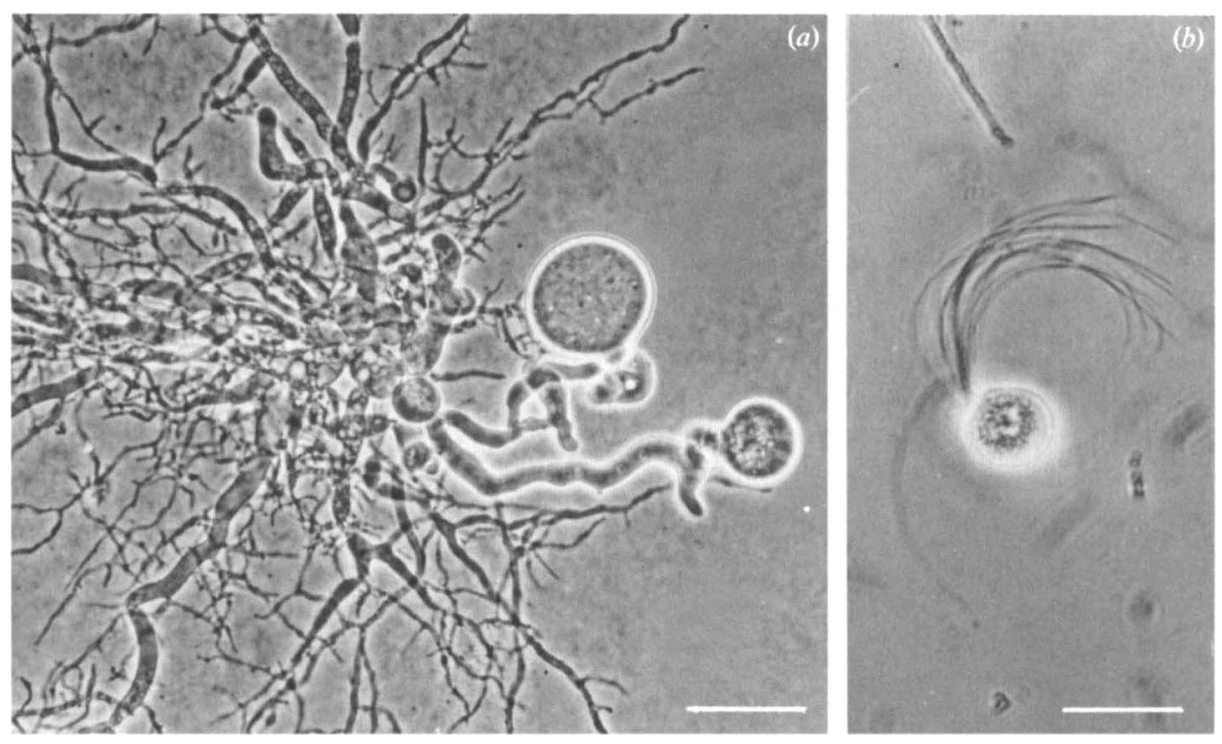

Fig. 1. Rhizomycelium and zoospore of LC2. (a) Rhizomycelium consisting of branched hyphae and sporangiophores with undifferentiated sporangia. Bar, $40 \mu \mathrm{m}$. (b) A polyflagellate zoospore of LC2. Bar, $20 \mu \mathrm{m}$.

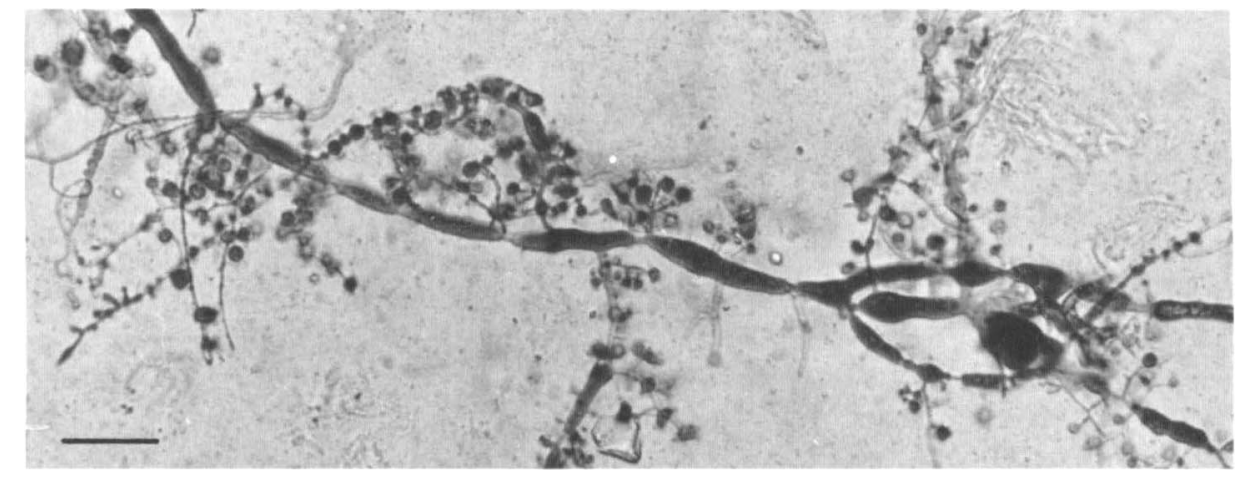

Fig. 2. Rhizomycelium of $R$. elegans showing both wide, constricted hyphae and narrow hyphae with lobed or disc-like structures. Bar, $20 \mu \mathrm{m}$.

possessing sporangia with acuminate tips and producing monoflagellate zoospores was mentioned by Phillips (1988), but insufficient details were given to determine if it is the same species as $R$. elegans. Fusiform sporangia with acuminate apices have also been observed in fungi colonizing grass fragments in the rumen of cattle and swamp buffaloes (Bauchop, 1979a; Ho et al., 1988b).

When pieces of mycelial mats of LL and LC2, with abundant undifferentiated sporangia after 3-4 d growth, were transferred into fresh liquid medium (GSM, CELB) or clarified rumen fluid, zoosporogenesis was observed to occur in many sporangia (Fig. $4 a-c$ ). Liberation of the zoospores occurred within 17-20 min of transfer. Maximum zoosporogenesis and liberation of zoospores occurred around $30-40 \mathrm{~min}$. Using a dissecting microscope, abundant polyflagellated zoospores were readily observed moving vigorously in the medium or close to the sporangium from which they had been released. Sporangia present in colonies growing in CAM and GAM in roll tubes also differentiated and released zoospores within $20 \mathrm{~min}$ of flooding with either fresh liquid medium (GSM, CELB) or clarified rumen fluid.

The age of sporangia at the time of transfer to fresh medium, or when flooded with fresh medium, was an important determinant of zoospore differentiation and liberation. Recently-formed sporangia differentiated and liberated zoospores most readily when transferred to, or flooded with, fresh medium. Older sporangia, i.e. those that were left for an additional $1-2 \mathrm{~d}$ in the original culture medium before transfer to fresh medium, did not differentiate to form zoospores when eventually transferred. Thus, transferring sporangia at the correct time and physiological stage appears to be essential to ensure abundant zoosporogenesis and liberation of zoospores. 

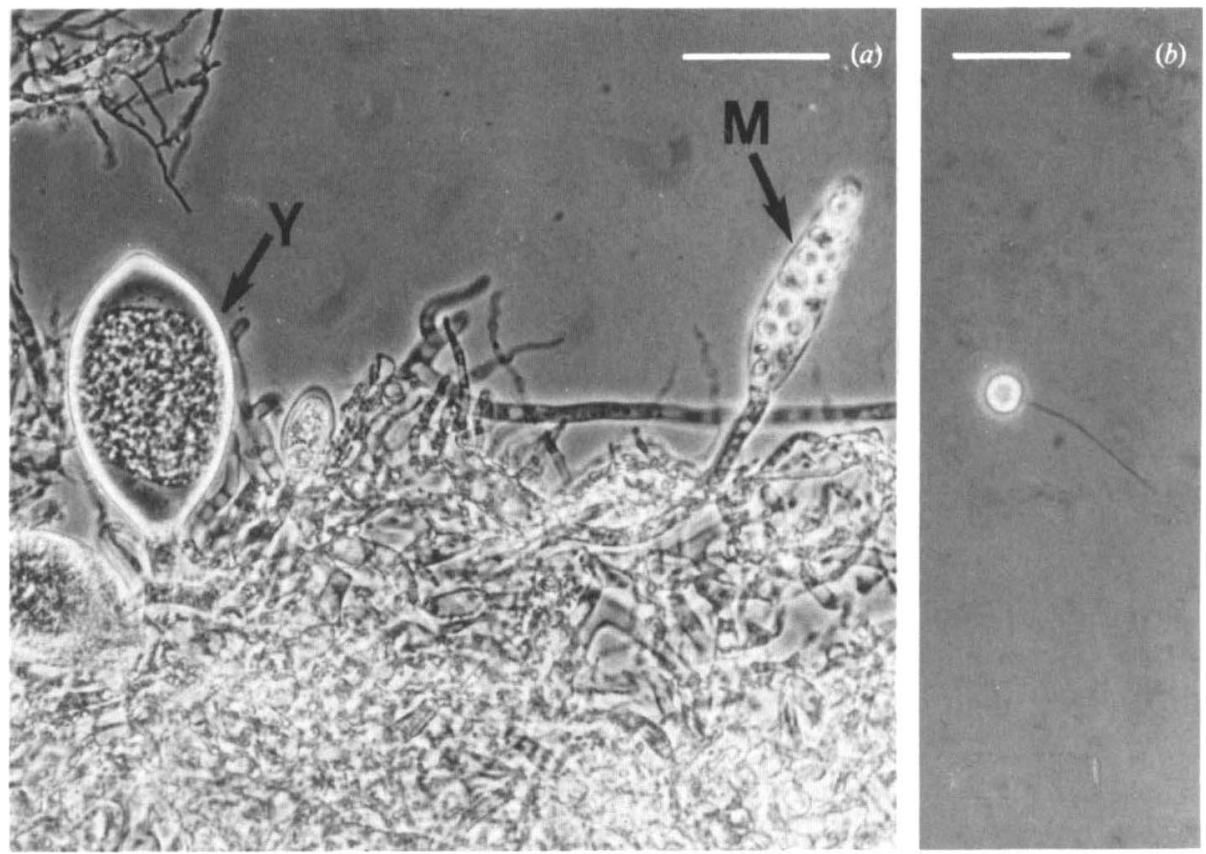

Fig. 3. Sporangium and zoospore of $R$. elegans. (a) A young fusiform sporangium ( $\mathrm{Y}$ ) and a mature sporangium (M) containing zoospores. Bar, $40 \mu \mathrm{m}$. (b) A monoflagellate zoospore. Bar, $20 \mu \mathrm{m}$.
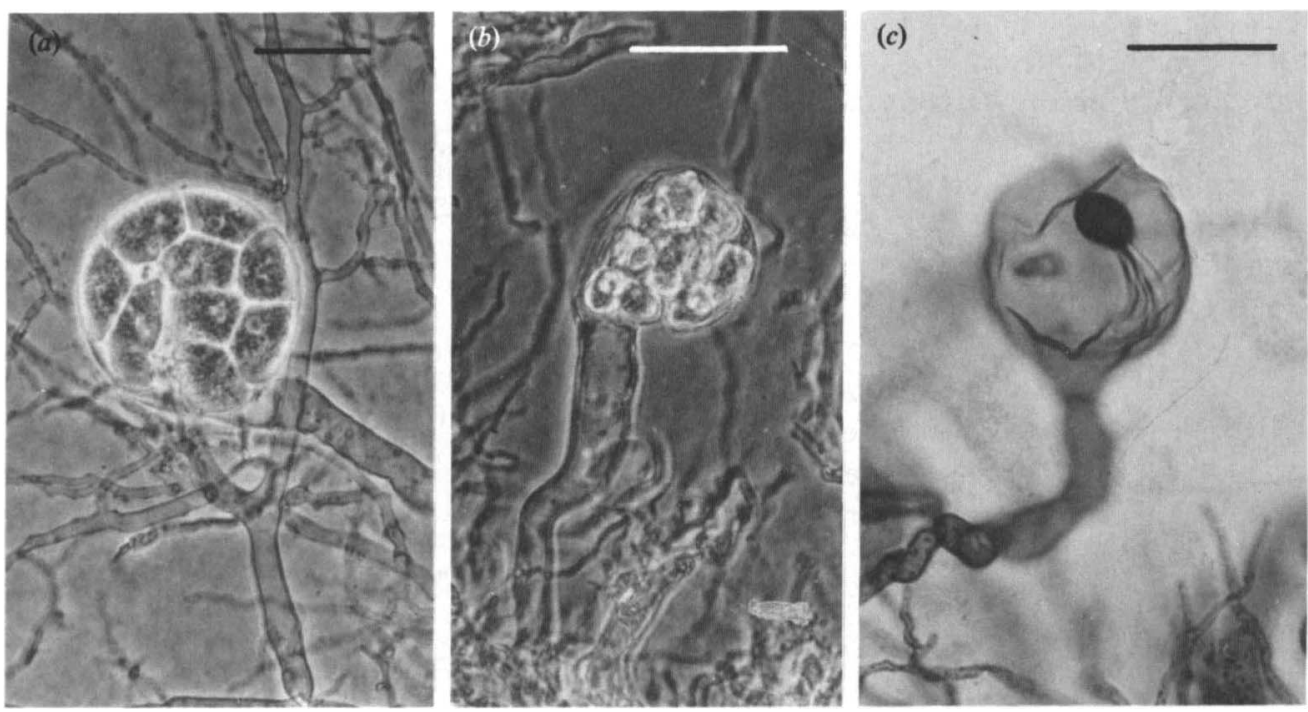

Fig. 4. Mature sporangia of LC2. (a) Contents of sporangium in the process of differentiating into zoospores; (b) sporangium containing zoospores; $(c)$ a single zoospore remaining in a sporangium after liberation of the other zoospores. Bars, $40 \mu \mathrm{m}$.

Colonies of $R$. elegans with abundant undifferentiated sporangia developed after $2-3 \mathrm{~d}$ growth in WSB or in CSM. When these were transferred to fresh GSM or clarified rumen fluid, zoosporogenesis (Fig. $3 a$ ) and liberation of zoospores again occurred within $30 \mathrm{~min}$. Zoosporogenesis and release of zoospores also occurred in sporangia which developed in $2 \mathrm{~d}$-old colonies growing on CAM when flooded with fresh GSM or clarified rumen fluid. As with LL and LC2, the age of the sporangia at the time of transfer was crucial and only newly-developed sporangia differentiated and released zoospores.

These results demonstrate that sporangia of the three polycentric rumen fungi LL, LC2 and $R$. elegans were 
induced to differentiate and release zoospore only when transferred to fresh media and when they were at the appropriate stage of development. Under these circumstances, conditions were provided for successful germination and development into mature thalli. Nutrient(s) that trigger zoosporogenesis may be depleted in media in which fungi have been grown for several days. Alternatively, fermentation end-products inhibitory to zoosporogenesis could have accumulated in the media.

With the monocentric fungi, Orpin (1977b) reported that in vivo zoosporogenesis of Neocallimastix frontalis, Piromonas communis and Sphaeromonas communis was induced by a water-soluble component in the diet of the host animal. One of these components was later found to be haem (Orpin \& Greenwood, 1986). For $N$. frontalis, maximum production of zoospores occurred 15-30 min after the onset of feeding (Orpin, 1975), but for Sphaeromonas and Piromonas, maximum production occurred about $1 \mathrm{~h}$ after feeding (Orpin, 1976 1977a). However, zoosporogenesis could not be induced by the addition of haem to $N$. frontalis grown in vitro in haemfree cultures, although partial zoosporogenesis $(<35 \%)$ did occur in response to addition of haem to $N$. frontalis grown in haem-limited cultures (Orpin \& Greenwood, 1986). Several haem-containing compounds were also found to induce zoosporogenesis and it was proposed that haem in synergism with other compounds in the diet induced abundant zoosporogenesis in the rumen (Orpin \& Greenwood, 1986).

The compounds responsible for induction of zoosporogenesis in polycentric fungi have not been investigated in this study. However, the marked contrast between monocentric and polycentric fungi in the degree of zoosporogenesis and zoospore release in vitro indicates fundamental differences. Unlike the monocentric fungi, zoospore production is not absolutely necessary for propagation of the polycentric fungi as they can be propagated by hyphae. The requirements for inducing zoosporogenesis of the polycentric fungi in vitro include sporangial development to the appropriate stage, the supply of zoosporogenic stimulants, and, possibly, removal of inhibitory fermentation products. Conditions for induction of zoosporogenesis in vitro may be different from those in vivo.

The present work has demonstrated a means of producing abundant zoosporogenesis and zoospore re- lease in rumen polycentric fungi; this will facilitate the systematic study of these anaerobic organisms.

\section{References}

AKIN, D. E. \& RigsBy, L. L. (1987). Mixed fungal populations and lignocellulosic tissue degradation in the bovine rumen. Applied and Environmental Microbiology 53, 1987-1995.

Akin, D. E., Borneman, W. S. \& Windham, W. R. (1988). Rumen fungi: morphological types from Georgia cattle and the attack on forage cell walls. BioSystems 21, 385-391.

BARR, D. J. S., Kudo, H., JAKOBER, K. D. \& CheNG, K. J. (1989). Morphology and development of rumen fungi: Neocallimastix sp., Piromonas communis and Orpinomyces bovis gen. nov., sp. nov. Canadian Journal of Botany 67, 2815-2824.

BAUCHOP, T. (1979a). Rumen anaerobic fungi of cattle and sheep. Applied and Environmental Microbiology 38, 148-158.

BaUCHOP, T. $(1979 b)$. The rumen anaerobic fungi: colonizers of plant fibre. Annales de Recherches Vétérinaires 10, 246-248.

BAUCHOP, T. (1989). Biology of gut anaerobic fungi. BioSystems 23, $53-$ 64.

Breton, A., Bernalier, A., Bonnemoy, F., Fonty, G., Gaillard, B. \& GouET, P. (1989). Morphological and metabolic characterization of a new species of strictly anaerobic rumen fungus: Neocallimastix joyonii. FEMS Microbiology Letters 58, 309-314.

Heath, I. B., Bauchop, T. \& Skipp, R. A. (1983). Assignment of the rumen anaerobe Neocallimastix frontalis to the Spizellomycetales (Chytridiomycetes) on the basis of its polyflagellate zoospore ultrastructure. Canadian Journal of Botany 61, 295-307.

Ho, Y. W., Abdullah, N. \& Jalaludin, S. (1988a). Penetrating structures of anaerobic rumen fungi in cattle and swamp buffalo. Journal of General Microbiology 134, 177-181.

Ho, Y. W., Abdullah, N. \& Jalaludin, S. (1988b). Colonization of guinea grass by anaerobic rumen fungi in swamp buffalo and cattle. Animal Feed Science and Technology 22, 161-171.

Ho, Y. W. Abdullah, N. \& Jalaludin, S. (1990). Ruminomyces elegans gen. et sp. nov., a polycentric anaerobic rumen fungus from cattle. Mycotaxon 38, 397-405.

HuNGaTe, R. F. (1969). A roll tube method for cultivation of strict anaerobes. Methods in Microbiology 3B, 117-132.

ORPIN, C. G. (1975). Studies on the rumen flagellate Neocallimastix frontalis. Journal of General Microbiology 91, 249-262.

ORPIN, C. G. (1976). Studies on the rumen flagellate Sphaeromonas communis. Journal of General Microbiology 94, 270-280.

ORPIN, C. G. (1977a). The rumen flagellate Piromonas communis: its life-history and invasion of plant material in the rumen. Journal of General Microbiology 99, 107-117.

ORPIN, C. G. $(1977 b)$. On the induction of zoosporogenesis in the rumen phycomycetes Neocallimastix frontalis, Piromonas communis and Sphaeromonas communis. Journal of General Microbiology 101, 181-189.

ORPIN, C. G. \& GReENwoOd, Y. (1986). Effects of haems and related compounds on growth and zoosporogenesis of the rumen phycomycete Neocallimastix frontalis H8. Journal of General Microbiology 132, 2179-2185.

Phillips, M. W. (1988). Unusual rumen fungi isolated from northern Australian cattle and water buffalo. In The Role of Protozoa and Fungi in Ruminant Digestion, pp. 247-249. Edited by J. V. Nolan, R. A. Leng \& D. I. Demeyer. Armidale: Penambul Books. 